\title{
Lagrangian Relaxation-Based Unit Commitment Considering Fast Response Reserve Constraints*
}

\author{
C. S. Chuang, G. W. Chang \\ Department of Electrical Engineering, National Chung Cheng University \\ Email: wchang@ee.ccu.edu.tw \\ Received September, 2012
}

\begin{abstract}
Unit commitment (UC) is to determine the optimal unit status and generation level during each time interval of the scheduled period. The purpose of UC is to minimize the total generation cost while satisfying system demand, reserve requirements, and unit constraints. Among the UC constraints, an adequate provision of reserve is important to ensure the security of power system and the fast-response reserve is essential to bring system frequency back to acceptable level following the loss of an online unit within a few seconds. In this paper, the authors present and solve a UC problem including the frequency-based reserve constraints to determine the optimal FRR requirements and unit MW schedules. The UC problem is solved by using Lagrangian Relaxation-based approach and compared with the actual system schedules. It is observed that favorable reserve and unit MW schedules are obtained by the proposed method while the system security is maintained.
\end{abstract}

Keywords: Unit Commitment; Fast Response Reserve; Frequency-based Reserve Constraints; Lagrangian Relaxation

\section{Introduction}

The unit commitment (UC) problem is to optimize hourly schedules of unit operation and minimize system operating costs for a given time interval. The constraints that the schedules must meet include system demand, reserve requirements, and unit constraints. Among the system constraints, the reserve is a crucial requirement for maintaining system frequency within the normal limits without any load shedding when the system experiences a contingency. Especially for an isolated power system, the system frequency is sensitive in responding to the load variation. Therefore, it is extremely important to schedule sufficient fast-response reserve (FRR) capacity to maintain the system security. The response time of FRR is usually the order of seconds to arrest the initial fall in frequency following the loss of any online generation unit. Therefore, the criterion of determining the FRR is difficult, since it varies from system to system. In [1] and [2], the load-frequency sensitivity index (LFSI) was used to assess the frequency drop following the loss of the largest online unit. However, this method still had to recalculate the reserve levels until the frequency constraint was met by an iterative procedure. In this paper, the frequency-based reserve constraints are considered in the UC problem. The LFSI and unit MW schedules are

\footnotetext{
*This work was supported in part by National Science Council of Taiwan, under grants NSC101-3113-P-110-001.
}

determined simultaneously. Then, the required FRR at each time step without violating the minimum system frequency is obtained. The UC problem including frequency reserve-based constraint is described and the problem is solved by using Lagrangian Relaxation-based approach. Simulation results and numerical experiences compared with the actual system are the reported.

\section{Problem Formulation}

The following gives the addressed UC problem formulation. More details can be found in [3] and [4].

\subsection{Objective Function}

The objective function is to minimize the operation cost, the cost of power purchase, and the compensation cost of violating the number of limit associated with independent power producers' (IPP) unit startup and shutdown.

$$
\begin{aligned}
F & =\sum_{t \in T} \sum_{i \in N}\left[f_{i}\left(p_{i}^{t}\right)+\left(y_{i}^{t} \times C_{u, i}\right)+\left(z_{i}^{t} \times C_{d, i}\right)\right] \\
& +\sum_{t \in T} \sum_{i \in N_{c}} \sum_{j=1}^{N_{T, i}}\left[c f_{i}\left(p_{c, i}^{t}\right)+\left(y_{c, i, j}^{t} \times C C_{u, i, j}\right)+\left(z_{c, i, j}^{t} \times C C_{d, i, j}\right)\right] \\
& +\sum_{t \in T} \sum_{i \in N_{p}}\left(\underline{p}_{p h, i} \times u_{i p, i}^{t}+\tilde{p}_{i p, i}^{t}\right) \times C_{p h, i}+\sum_{i \in N_{p}}\left(T_{y z, i}-N_{s s, i}\right) \times C_{m, i}
\end{aligned}
$$

where $f_{i}$ and $c f_{i}$ are the fuel cost functions for the thermal and combined-cycle units; $C_{u, i}, C_{d, i}, C C_{u, i, j}$, and $C C_{d, i, j}$, are 
the unit startup and shutdown costs; $C_{p h, i}$ and $C_{m, i}$ are the IPP power purchase and compensation costs; $-p h, i$ is the minimum power purchase of IPP unit; $N_{s s, i}$ is the maximum number of startups/shutdowns of IPP unit. $T$ is the set of scheduled time steps; $N, N_{c}$, and $N_{p}$ are the sets of thermal, combined-cycle, and IPP units. $N_{T, i}$ is the configuration number of the combined-cycle unit. The last term of (1) represents the penalty cost for the $i$-th IPP unit. When the power company dispatches the IPP units and the number of startups /shutdowns exceeds the allowed maximum number, the power company pays the penalty cost to the IPP.

\subsection{System Constraints}

1) Power Balance Constraints

$$
\begin{aligned}
& \sum_{i \in N} p_{i}^{t}+\sum_{i \in N_{c}} p_{c, i}^{t}+\sum_{i \in N_{p}} p_{i p, i}^{t}+\sum_{k \in K_{r}} \sum_{i \in N_{p, k}} p_{s, i, k}^{t}=D^{t}, \\
& \forall t \in T
\end{aligned}
$$

where $K_{r}$ is the set of reservoirs and $N_{p, k}$ is the set of pumped-storage units associated with reservoir $k$.

\section{2) Reserve Constraints}

The reserve constraints include FRR, $\mathrm{SR}_{10}$, and $\mathrm{OR}_{30}$ requirements. The FRR is supplied by synchronized pumped-storage units. The $\mathrm{SR}_{10}$ is provided by nonsynchronized pumped-storage units. The source of $\mathrm{OR}_{30}$ is composed of non-synchronized combined-cycle units. The amounts of the required $\mathrm{SR}_{10}$ and $\mathrm{OR}_{30}$ are preset parameters, while the required FRR in (3) is not constant through the study period and it varies according to the unit MW schedules. The calculation of the FRR is described in section III.

$$
\begin{aligned}
& \sum_{k \in K_{r}} \sum_{i \in N_{p, k}} u_{p g, i, k}^{t} \bar{p}_{s, i, k}-p_{s, i, k}^{t} \geq F R R^{t}, \quad \forall t \in T \\
& \sum_{k \in K_{r}} \sum_{i \in N_{p, k}}\left(1-u_{p g, i, k}^{t}-u_{p p, i, k}^{t}\right) \bar{p}_{s, i, k} \geq S R_{10}^{t}, \quad \forall t \in T \\
& \sum_{i \in N_{c}}\left(1-\sum_{j=1}^{N_{T, i}} u_{c, i, j}^{t}\right) \bar{p}_{c, i} \geq O R_{30}^{t}, \quad \forall t \in T
\end{aligned}
$$

where $\bar{p}_{s, i, k}$ and $\bar{p}_{c, i}$ are the maximum MW outputs for the pumped-storage and combined-cycle units, respectively.

\subsection{Unit Model and Constraints}

The considered units include thermal units, combinedcycle units, pumped-storage units, and IPP units. The constraints associated with the thermal units and combined-cycle units a can be found in [3] and [4].

In the system under study, each IPP unit has signed a power purchase contract with the power company, where the power company must purchases and dispatches the
MW output of each IPP unit. The power purchase from an IPP unit $i$ and the sum of contractual number of hours, $H_{i}$, of the associated unit must be online for company's dispatch are given by (6) and (7), respectively.

$$
\begin{gathered}
p_{i p, i}^{t}=\underline{p}_{p h, i} \times u_{i p, i}^{t}+\tilde{p}_{i p, i}^{t} \leq \bar{p}_{p h, i}, \quad \forall t \in T, i \in N_{p} \\
\sum_{t \in T} u_{i p, i}^{t} \geq H_{i}, \forall i \in N_{p} .
\end{gathered}
$$

where $\underline{p}_{p h, i}$ and $\bar{p}_{p h, i}$ are the minimum and maximum power purchases from IPP unit $i$, respectively. The number of startups and shutdowns for the i-th IPP unit is also limited not exceeding the contractual total number.

2) Pumped-storage Unit Model and Constraints

As illustrated in [4], the I/O curve for a specific head level is approximated by a two-segment linear curve in the generating mode; in the pumping mode, a discrete point is modeled to represent the pumping status at full load. The pumping MW capacity must be greater than the FRR requirement during off-peak load period, hours 1 to $N_{o p k}$, to maintain the system security and reliability. Then, the Must-Pumping Unit Constraint is considered and is given in (8).

$$
\sum_{k \in K_{r}} \sum_{i \in N_{p, k}} P_{p, i, k} \times u_{p p, i, k}^{t} \geq F R R^{t}, \quad \forall t \in 1 \ldots N_{o p k}
$$

where $P_{p, i, k}$ is the pumping power of the pumped- storage unit $i$ associated with reservoir $k$.

\section{Load-frequency Sensitivity Index and Frequency-Based Reserve Constraint}

The system frequency variation during a contingency is highly related to the system load characteristics and is difficult to measure. For simplification, the load-frequency sensitivity index (LFSI) is used to assess the load behavior following the loss of an online unit [1,2]. A brief interpretation of the proposed LFSI is described below

\subsection{Load-frequency Sensitivity Index}

The LFSI at time step $t$, labeled by $\eta^{t}$ in (9), can be calculated by using the recorded system frequency during actual contingencies.

$$
\eta^{t} \approx \Delta P_{G}^{t} / \Delta f_{S}
$$

where $\Delta P_{G}^{t}$ is the amount of MW generation loss (in percentage of the system load at time step $t$ ) and $\Delta f_{s}$ is the system frequency drop following the loss of an online unit.

The calculation of LFSI of (9) depends on the system load characteristics, the operation mode of pumped- storage units, and the trend of the load variation with time $\left(d D^{t} / d t\right)$. In this paper the authors adopt and improve a 
statistical approach employed in [2] to determine the mean value, $\mu$, and the standard deviation, $\sigma$, of LFSI for three time intervals (hours 1-8, 9-16, and 17-24) in one day based on the historical data. The LFSI is then calculated for each time interval according to the new determinative criteria stated below.

\subsubsection{With Pumping Load}

In the light load period, the pumped -storage units are typically operated in pumping mode. Therefore, the pumping load can be shed quickly to replace the MW loss when the system loss an online unit. The system frequency deviation is thus relatively smaller. Then, the LFSI is larger and the required FFR is less than that without pumping load, regardless of the load variation trend. For both load variation trends, the LFSI is set to be $\mu+\sigma$.

\subsubsection{Without Pumping Load}

When the loss of a generating unit occurs not in the light load period, the percentage of MW generation loss to the total system load is smaller than that occurs in the other time period. The system frequency deviation will be larger under increasing load (i.e. $d D^{t} / d t>0$ ) than under decreasing load (i.e. $\left.d D^{t} / d t<0\right)$. The required FRR for the increasing load case must cover both the MW generation loss and the incremental system load; it is more than that of the decreasing case. Therefore, the LFSI for the increasing load case is set to be $\mu-\sigma$ to supply more FRR. For the case of decreasing load, the LFSI is set to be $\mu$.

\subsection{Adaptive LFSI and Frequency-Based Reserve Constraint}

In this paper, the concept of the adaptive LFSI is proposed according to the aforementioned pumping status and the load variation trends. The determination of required FFR is included in the UC problem. The optimal unit MW schedules and the calculated LFSI are determined at each time step after the UC problem is solved. To model the described four criteria with and without pumping load and the load characteristics, the expression of the proposed adaptive LFSI is shown in (10).

$$
\eta^{t}=\mu^{t}+\left[P M P S^{t}+\left(P M P S^{t}-1\right) \cdot L V^{t}\right] \cdot \sigma^{t}
$$

where $P M P S^{t}$ and $L V^{t}$ are the pumped load index (binary variable) and the load variation index (binary parameter) at time step $t$, respectively. $L V^{t}$ is pre-determined according to the load forecast information of the UC problem. If $d D^{t} / d t \geq 0$, then $L V^{t}=1$. Otherwise, $L V^{t}=0$. $P M P S^{t}$ is defined as

$$
P M P S^{t}= \begin{cases}1, & \text { system including pumping load, } \\ 0, & \text { otherwise. }\end{cases}
$$

and is expressed by the following explicit constraint:

$$
\left(\sum_{k=1}^{K_{r}} \sum_{i=1}^{N_{p, k}} u_{p p, i, k}^{t}\right) / \mathrm{L} \leq \text { PMPS }^{t} \leq \sum_{k=1}^{K_{r}} \sum_{i=1}^{N_{p, k}} u_{p p, i, k}^{t}, \forall t \in T
$$

where $\mathrm{L}$ is a sufficiently large positive number. Therefore, the $P M P S^{t}$ will be determined according to the pumped-storage unit states at each time step and the adaptive LFSI in (10) will be adjusted according the $P M P S^{t}$ rather than a predetermined parameter. In order to supply the appropriate FRR, a way to determine the minimum FRR is to set the recovery frequency, $f_{\text {rec }}$, being slightly greater than the allowable minimum frequency, $f_{\min }$. Such frequency-based reserve constraint is given in (13).

$$
F R R^{t} \geq P_{G m x}-\left(f_{s}-f_{\min }\right) \times \eta^{t} \times D^{t}
$$

where $P_{G m x}$ is the pre-determined MW generation of the largest online unit; $f_{\text {rec }}$ is the recovery frequency after starting FRR; $f_{s}$ is the system nominal frequency (i.e. $60 \mathrm{~Hz}$ ) and $f_{\min }$ is the specified minimum frequency.

\section{Implementation and Solution Technique}

In this paper, the proposed UC problem is solved by the LR-based approach. The coupling constraints of (2)-(5), (8), and (12) are relaxed and added to the objective function. The Lagrangian function then becomes (14).

$$
\begin{aligned}
& L=F+\sum_{t=1}^{T} \lambda^{t}\left[D^{t}-\sum_{i=1}^{N} p_{i}^{t}-\sum_{i=1}^{N_{c}} p_{c, i}^{t}-\sum_{i=1}^{N_{p}} p_{i p, i}^{t}-\sum_{k=1}^{K_{r}} \sum_{i=1}^{N_{p, k}} p_{s, i, k}^{t}\right] \\
& +\sum_{t=N_{\text {opk }}+1}^{T} \mu_{F R R}^{t}\left[F R R^{t}-\sum_{k=1}^{K_{r}} \sum_{i=1}^{N_{p, k}} u_{p g, i, k}^{t} \bar{p}_{s, i, k}-p_{s, i, k}^{t}\right] \\
& +\sum_{t=1}^{T} \mu_{S R 10}^{t}\left[S R_{10}^{t}-\sum_{k=1}^{K_{r}} \sum_{i=1}^{N_{p, k}}\left(1-u_{p g, i, k}^{t}-u_{p p, i, k}^{t}\right) \bar{p}_{s, i, k}\right] \\
& +\sum_{t=1}^{T} \mu_{O R 30}^{t}\left[\text { OR } R_{30}^{t}-\sum_{i=1}^{N_{c}}\left(1-\sum_{j=1}^{N_{T, i}} u_{c, i, j}^{t}\right) \bar{p}_{c, i}\right] \\
& +\sum_{t=1}^{N_{o p k}} \mu_{o p k}^{t}\left[F R R^{t}-\sum_{k=1}^{K_{r}} \sum_{i=1}^{N_{p, k}} P_{p, i, k} u_{p p, i, k}^{t}\right] \\
& +\sum_{t=1}^{T} \mu_{P M_{-}}^{t}\left[\frac{1}{L} \sum_{k=1}^{K_{r}} \sum_{i=1}^{N_{p, k}} u_{p p, i, k}^{t}-P M P S^{t}\right] \\
& +\sum_{t=1}^{T} \mu_{P M_{+}}^{t}\left[P M P S^{t}-\sum_{k=1}^{K_{r}} \sum_{i=1}^{N_{p p, k}} u_{p p p, i, k}^{t}\right] \\
& + \text { "relaxation of reservoir volume limits" }
\end{aligned}
$$

where $\lambda^{t}, \mu_{F R R}^{t}, \mu_{S R 10}^{t}, \mu_{O R 30}^{t}$, and $\mu_{o p k}^{t}$ are Lagrange multipliers associated with (2)-(5), and (8), respectively. The Lagrange multipliers, $\mu_{P M_{-}}^{t}$ and $\mu_{P M_{+}}^{t}$, 
are associated with the lower and upper bounds of (12), respectively. The last term of (14) is for the relaxation of the reservoir volume limits of the pumped- storage hydro units and the corresponding constraints can be found in [4]. Major steps of the LR solution procedure are summarized as follows:

1) Initialize the Lagrange multipliers.

2) Solve the sub-problems by minimizing the Lagrangian function subjecting to local constraints of each subproblem.

3) Check if all system reserve constraints are satisfied at each time step according to the solutions obtained at Step 2. If all reserve constraints are satisfied, proceed to the next step; otherwise, go to Step 6 .

4) Apply the heuristic method of [5] for pumped- storage hydro units to obtain a feasible solution (i.e. unit state and generation or pumping MW output) based on the dual solutions. By using the adopted heuristic method, the reservoir volume limits are guaranteed to be satisfied at each time step. Since some pumped-storage hydro units are approximately identical in the study system, if the selected unit needs to reduce the generation or increase the pumping MW output, one of the identical units will be randomly selected during the heuristic solution process. When the feasible solution is obtained, proceed to the next step.

5) Perform economic dispatch and then check if the system convergence criterion of $1 \%$ duality gap is satisfied. If the assigned duality gap is met, stop the procedure; otherwise, proceed to the next step.

6) Update the Lagrange multipliers by using the subgradient method and return to Step 2.

\section{Results}

The proposed problem model has been implemented and tested with actual system data. In the study, the test system consists of 29 thermal units, 21 combined-cycled units, 13 IPP units, and 2 pumped-storage hydro plants including 10 units (6 units in Plant A and 4 units in Plant B) for one-day simulation. The amount of $\mathrm{SR}_{10}$ is set to be $1000 \mathrm{MW}$ and the amount of the $\mathrm{OR}_{30}$ is set to be $9 \%$ of the total system load. The minimum frequency of (13) is $59.7 \mathrm{~Hz}$. The largest online unit MW generation of (13) is $980 \mathrm{MW}$. The simulated results compared with the actual system schedules are shown in Figure 1 and Table 1.

Figure 1 depicts the MW schedule obtained by the proposed method and the load curves. By observing Table 1, it is seen that the scheduled FRR obtained by proposed method is less than the actual system schedule. Also, the recovery frequency can be maintained at allowable minimum frequency of $59.7 \mathrm{~Hz}$ to save the operation cost.

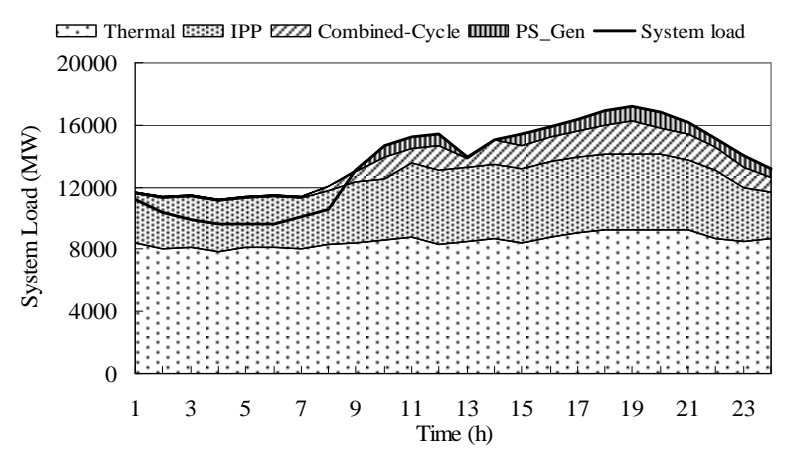

Figure 1. MW schedule obtained by LR and load curves.

Table 1. Comparison of FRR (MW) and Recovery Frequency (Hz).

\begin{tabular}{|c|c|c|c|c|}
\hline Hr. & $\begin{array}{c}\text { FRR } \\
\text { [Actual-Sys.] } \\
\text { (MW) }\end{array}$ & $\begin{array}{c}f_{\mathrm{rec}} \\
(\mathrm{Hz})\end{array}$ & $\begin{array}{c}\text { FRR_LR } \\
(\mathrm{MW})\end{array}$ & $\begin{array}{c}f_{\text {rec }} \\
(\mathrm{Hz})\end{array}$ \\
\hline 1 & 567.60 & 59.78 & 308.24 & 59.70 \\
\hline 2 & 523.08 & 59.79 & 317.68 & 59.70 \\
\hline 3 & 529.54 & 59.79 & 327.04 & 59.70 \\
\hline 4 & 534.93 & 59.79 & 334.85 & 59.70 \\
\hline 5 & 538.20 & 59.79 & 339.60 & 59.70 \\
\hline 6 & 536.17 & 59.79 & 336.65 & 59.70 \\
\hline 7 & 521.32 & 59.79 & 315.13 & 59.70 \\
\hline 8 & 594.37 & 59.82 & 319.73 & 59.70 \\
\hline 9 & 710.15 & 59.80 & 269.85 & 59.70 \\
\hline 10 & 757.34 & 59.85 & 537.68 & 59.70 \\
\hline 11 & 782.65 & 59.87 & 522.90 & 59.70 \\
\hline 12 & 785.54 & 59.87 & 521.21 & 59.70 \\
\hline 13 & 542.92 & 59.82 & 231.63 & 59.70 \\
\hline 14 & 527.67 & 59.82 & 205.53 & 59.70 \\
\hline 15 & 782.55 & 59.87 & 522.95 & 59.70 \\
\hline 16 & 793.07 & 59.88 & 516.81 & 59.70 \\
\hline 17 & 808.10 & 59.89 & 508.03 & 59.70 \\
\hline 18 & 824.70 & 59.90 & 498.34 & 59.70 \\
\hline 19 & 834.63 & 59.91 & 492.54 & 59.70 \\
\hline 20 & 642.97 & 59.84 & 337.03 & 59.70 \\
\hline 21 & 625.81 & 59.83 & 354.19 & 59.70 \\
\hline 22 & 604.44 & 59.81 & 375.56 & 59.70 \\
\hline 23 & 557.47 & 59.82 & 416.94 & 59.70 \\
\hline 24 & 527.79 & 59.74 & 452.21 & 59.70 \\
\hline
\end{tabular}

\section{Conclusions}

In this paper, the concept of LFSI is introduced and the new determinative criteria for the LFSI are proposed. The accurate minimum FRR limit and the unit MW schedules can be determined simultaneously when solving the UC problem and the optimal MW schedule is achieved. Test results obtained by the LR method are also compared with actual system schedules. It shows that the proposed method yields less cost of unit MW 
generation and FFR schedules while the system security is maintained.

\section{REFERENCES}

[1] C. C. Wu and N. Chen, "Online Methodology to Determine Reasonable Spinning Reserve Requirement for Isolated Power system," IEE Proc.-Gene. Transm. and Distri., Vol. 150, No. 4, July 2003, pp. 455-461.

[2] C. C. Wu and N. Chen, "Frequency-based Method for Fast-response Reserve Dispatch in Isolated Power System,” IEE Proc.-Gene. Transm. and Distri., Vol. 151, No. 1, Jan. 2004, pp. 73-77.

[3] G. W. Chang, C. S. Chuang and T. K. Lu, “A Simplified Combined-cycle Unit Model for Mixed Integer Linear

\section{Nomenclature}

Listed below are notations of variables used in the UC problem formulation throughout the paper.

$p_{i}^{t}=\mathrm{MW}$ output of thermal unit $i$ at time step $t$

$y_{i}^{t}=$ Startup state of thermal unit $i$ at time step $t$ (binary)

$z_{i}^{t}=$ Shutdown state of thermal unit $i$ at time step $t$ (binary)

$p_{c, i}^{t}=\mathrm{MW}$ output of combined-cycle unit $i$ at time step $t$ $y_{c, i, j}^{t}=$ Startup state of $j$-th configuration of combined-cycle unit $i$ at time step $t$ (binary)
Programming-based Unit Commitment," Proceedings of the 2008 IEEE PES General Meeting, Pittsburgh, PA, USA, July 2008.

[4] G. W. Chang, M. Aganagic, J. G. Waight, J. Medina, T. Burton, S. Reeves and M. Christoforidis, "Experiences with Mixed Integer Linear Programming Based Approaches on Short-term Hydro Scheduling," IEEE Transactions on Power Systems, Vol. 16, No. 4, 2001, pp. 743-749.doi:10.1109/59.962421

[5] X. Guan, P. B. Luh, H. Yan and P. Rogan, "Optimization-based Scheduling of Hydrothermal Power Systems with Pumped-storage Units," IEEE Transactions on Power Systems, Vol. 9, No. 2, 1994, pp. 1023-1031.doi:10.1109/59.317641

$z_{c, i, j}^{t}=$ Shutdown state of $j$-th configuration of combined-cycle unit $i$ at time step $t$ (binary)

$u_{i p, i}^{t}=$ on/off states of IPP unit $i$ at time step $t$ (binary)

$p_{i p, i}^{t}=$ MW purchase of IPP unit $i$ at time step $t$

$\tilde{p}_{i p, i}^{t}=\mathrm{MW}$ purchase of the segment of the I/O curve of IPP unit $i$ at time step $t$

$T_{y z, i}=$ Total number of startups/shutdowns of IPP unit $i$

$F R R^{t} \quad=$ fast-response reserve at time step $t$ (MW) $u_{p p, i, k}^{t}=$ on/off pumping state of the pumped-storage unit $\mathrm{i}$ within reservoir $\mathrm{k}$ at time step $\mathrm{t}$ 